\title{
Anti-PD-1/Anti-LAG-3 DART Protein MGD013
}

National Cancer Institute

\section{Source}

National Cancer Institute. Anti-PD-1/Anti-LAG-3 DART Protein MGD013. NCI Thesaurus.

Code 143957.

An Fc-bearing, humanized antibody-like protein that specifically recognizes the immune checkpoint molecules programmed cell death 1 (PD-1; PD1; PDCD1; CD279;

Programmed Death 1) and lymphocyte activation gene-3 (LAG-3; LAG3; CD223), with potential T-lymphocyte immunomodulatory and antineoplastic activities. Upon administration, the anti-PD-1/anti-LAG-3 dual-affinity re-targeting (DART) protein MGD013 specifically binds to both PD-1 and LAG-3, which are both expressed on T-cells. The dual blockade of the PD-1 and LAG-3 pathways enables potent activation of a cytotoxic T-lymphocyte (CTL)-mediated immune response against tumor cells. PD-1 and LAG-3 play key roles in suppressing T-cell activation. 